\title{
CONSIDERACIONES \\ SOBRE LAS CARTAS DE SANTA CATALINA DE SIENA \\ A LAS MUJERES DE SU TIEMPO, Y SU RECEPCIÓN EN ESPAÑA
}

\author{
MONTSERRAT CASAS NADAL \\ Universitat de Barcelona
}

A Regina Sáinz de la Maza, a su memoria y a su presencia

\section{SUMARIO}

0. Introducción.- 1. Características generales de las cartas: Protocolo de las cartas.- 2. Las cartas a las mujeres de su tiempo: a) Las cartas a las reinas. b) Cartas a las damas nobles. c) Cartas a las mujeres de su entorno familiar. d) Cartas dirigidas a sus discípulas. e) Cartas dirigidas a las terciarias dominicas o "mantellate". f) Cartas dirigidas a monjas o comunidades monásticas del centro de Italia. g) Cartas a diversas mujeres o grupos de mujeres de procedencia y ocupaciones muy diversas.- 3 . La recepción/traducción de las epistolas en España.

\section{INTRODUCCIÓN ${ }^{1}$}

De toda la obra literaria de santa Catalina de Siena, destacan por su alto significado humano y teológico, así como por su valor histórico, las Epístolas que, en número de 381 , dirigió a papas, cardenales y prelados, sacerdotes, frailes y monjes, condotieros, gobernantes de ciudades, artistas,

\footnotetext{
'Este trabajo se ha llevado a cabo en el marco del proyecto n PB94-0902 de la DGICYT

"Anuario de Lstudios Medievales". 28 (1998)
} 
juristas, médicos, familiares, discípulos, y a numerosas y variadas mujeres de su época, que jugaron papeles muy diversos dentro de la sociedad italiana de su tiempo, entre los años 1365 y 1380.

Las cartas de Catalina no sólo incidieron - con mayor o menor fortuna- en sus contemporáneos sino que han acompañado generaciones y generaciones de lectores, y gozaron de un alto nivel de popularidad dentro de la tradición literaria y lingüística italiana ${ }^{2}$.

Dada la complejidad y riqueza de estas cartas es imposible analizarlas todas ellas con cierto detalle, por este motivo me limito a unas consideraciones sobre las que van dirigidas a las mujeres. En cualquier caso, no puede olvidarse que las cartas se escriben en un preciso contexto histórico, iniciado con la convocatoria de la gran cruzada contra los otomanos ${ }^{3}$ - primero por Urbano V (1365) y después por Gregorio XI (1371) - , en medio de la crisis eclesiástica derivada de la larga estancia del papado en la ciudad de Aviñón y los inicios del Cisma de Occidente, tras la elección de Urbano VI en Roma y de Clemente VII en Aviñón. Catalina fue testigo presencial de todos estos hechos, defendió a ultranza la legitimidad del pontífice Urbano VI frente a reyes y cardenales, y observó en primera línea la no menos importante descomposición del mundo de los Comunes de la Italia central ${ }^{4}$. Éstos y muchos otros acontecimientos de su época, condicionaron y se reflejaron en la actividad epistolar de Catalina.

Una cuarta parte de las cartas de la santa tuvieron como destinatarias a mujeres, ya fuesen a título individual o como colectivo. Del conjunto de cartas consideradas como obra auténtica cateriniana pueden distinguirse: a)

\footnotetext{
${ }^{2}$ Adriano ProSPERI, Lettere spirituali, en "Donne e fede. Santità e vita religiosa in Italia", a cura di Lucetta SCARAFFIA e Gabriella ZANI, Laterza, Roma-Bari, 1994, pp. 227-251.

${ }^{3}$ Sobre el papel desempeñado por Catalina de Siena en la campaña de propaganda a favor de la cruzada, vid. Franco CARDINI, L'idea de Crociata in Santa Caterina da Siena, en "Atti del Simposio Internazionale Cateriniano-Bernardiano (Siena, 17-20 aprile 1980)", Accademia Senese degli Intronati, Siena, 1982, pp. 57-87.

${ }^{4}$ Catalina insistió, con todos los medios a su alcance, en conseguir el retorno de los papas a la Ciudad Eterna, sede de Pedro. La tradición piadosa ha considerado el regreso de los pontífices a Roma como obra casi personal de Catalina. Vid. Paul RENUCCI, La cultura, en "Storia d'Italia", diretta da R. Romano e C. Vivanti, vol. II, "Dalla caduta dell'Impero romano al secolo XVIII", Einaudi, Torino, 1974, pp. 1203-1204.

Sobre la actitud de Catalina ante la nueva realidad de los Comunes, es altamente significativa la carta dirigida al gonfalonier y a los priores de Florencia: «i signori naturali sono fatti tirani. Al petto del Comune non si nutricano i sudditi con giustizia né carità fraterna; ma ciascuno con falsità e bugie attende al bene proprio particolare, e non al bene universale Ognuno cerca la signoria per sé, e non il buon stato e reggimento della cittàm [337].
} 
las ocho dirigidas a reinas; b) 29 dirigidas a grandes damas de la aristocracia italiana; c) siete destinadas a mujeres de su estrecho círculo familiar, entre ellas cuatro a monna Lapa, su madre; d) siete dirigidas a sus discípulas, mayoritariamente a monna Alessa; e) 17 para las mantellate o terciarias de la orden dominicana; f) 17 a monjas o comunidades monásticas del centro de Italia; g) 13 a diversas mujeres o grupos de mujeres de procedencia y ocupaciones muy diversas, entre las cuales figura una meretriz y una penitente.

\section{CARACTERÍsticas gENERALES DE LAS CARTAS}

Catalina de Siena propone a través de su Epistolario - al igual que en los Diálogos y en la Vida, escrita por fray Raimundo de Capua- un modelo activo y heroico de espiritualidad femenina. Además, demuestra, a través de las cartas, la altísima conciencia de su acción apostólica; se ve a sí misma enviada por Dios para llevar a cabo un vasto plan de reforma religiosa tanto en las almas singulares como en la sociedad de su época ${ }^{5}$. Catalina se considera investida de un "primado" de servicio al frente de la Iglesia; esta especial consagración y misteriosa investidura se reflejan en el uso - casi burocrático- de titulaciones similares a las de los pontífices: «Catarina, serva dei servi di Dio», «Io, Catarina, serva e schiava de'servi di Gesù Cristo» [86, 217, 144], «Io Catarina, serva inutile di Gesù Cristo» [31]. De ahí que los escritos de Catalina - especialmente las Cartas - asuman la forma y tono de una auténtica predicación, del magisterio y de la amonestación. El tono exquisitamente maternal y humano que acompaña siempre la acción apostólica y la necesidad de comunicar la experiencia mística debieron de beber en las abundantes y continuadas lecturas de Vidas de santos y del breviario, y el contacto constante y directo con la comunidad del convento de Santo Domingo de Siena ${ }^{6}$.

No obstante, no aparece en el Epistolario una auténtica y concreta dirección de conciencias. Sus consejos -o los que marcaron su confesor y

\footnotetext{
168.

${ }^{5}$ Giovanni GeTto, Letteratura religiosa del Trecento, Sansoni Editore, Firenze, 1967, p.

${ }^{6}$ E. Dupré Theseider, Caterina da Siena, "Dizzionario biografico degli italiani", vol. 22 , Istituto della Enciclopedia Italiana, Roma, 1979, p. 373-374.
} 
director espiritual, fray Raimundo de Capua ${ }^{7}$ - se presentan harto vagos e imprecisos, cargados de exhortaciones genéricas que bien podrían estar dirigidas a toda la comunidad de creyentes, y no a personas con problemas concretos.

El tema de la vida espiritual prevalece en todas las epístolas, de suerte que no siempre es fácil encontrar diferencias y connotaciones singulares entre las diversas cartas. El mundo interior de las virtudes y de las pasiones, vivido dramáticamente, constituye el centro de interés de Catalina, repetido y reiterado en las diversas epístolas, hasta el punto que se ha podido afirmar que los protocolos de cada carta constituyen el elemento externo diferenciador ${ }^{8}$.

De todas formas el lenguaje de las epístolas de Catalina, además de una profunda carga sentimental, conlleva una clara voluntad exhortatoria que brolla de un complejo fondo doctrinal-didascálico". En este sentido pueden presentarse como ejemplos significativos las cartas dirigidas a Nanna di Benincasa [23], a la que ofrece el ejemplo y modelo evangélico de las vírgenes prudentes «le quali avevano le lampane coll'olio ed eravi il lume dentro»; o bien el de la carta dirigida a sor Eugenia, del monasterio de Santa Agnese de Montepulciano sobre las tres formas de oración [26]; e incluso los tres votos de pobreza, obediencia y castidad, calificados como las "tre colonne" de la vida religiosa, en carta dirigida a la priora y monjas de Santa Maria delle Vergini y a la priora de San Giorgio de Perugia [217].

Otra de las características peculiares de las epístolas caterinianas es la falta de preocupación por el uso de una exquisita corrección literaria; prefiere la lengua hablada a una expresión preciosista y opta por la naturalidad con que emerge su inspiración. El uso de expresiones metafóricas simples, de fácil comprensión y próximas a la cultura de la mujer de su entorno, aparece como hecho reiterativo y recurso frecuente en sus cartas:

\footnotetext{
${ }^{7}$ Fray Raymundo de Capua, además de ser uno de los más fervientes admiradores y defensores de la espiritualidad de Catalina de Siena, y celoso reformador de su orden, fue el encargado de velar y controlar la "ortodoxia" de la santa, a partir del momento en que la declarada desconfianza de los dominicos les llevó a convocarla al capítulo general de la orden. celebrado en Florencia. en 1374. Guido BALDASSARI, Letteratura devota, edificante e morale. en "Storia della Letteratura Italiana, vol.II, "Il Trecento", Salerno Editrice, Roma. 1995. pp. 292-293.

${ }^{8}$ Giovanni GetTo, Letteratura religiosa. p. 169

${ }^{9}$ Giovanni Getro. Letteratura religiosa, p. 172

También Giorgio PETROCCHI, La religiositì, en "Letteratura Italiana", diretta da A. Asor Rosa, vol. V, "Le questioni", Einaudi. Torino, 1986, pp. 125-168.
} 
Así resultan expresiones como "il latte dell'orazione", "la fonte della pietà", "il coltello dell'odio". "il giardino della santa Chiesa", "il fuoco della divina carità" o "il cane della coscienza". Un tal recurso, utilizado de forma un tanto reiterativa e incluso poco variada, ha llevado a algunos críticos a ver en estas expresiones de la santa la monotonía e incluso el cansancio que se advierte en la lectura continuada de las epístolas.

Debe tenerse en cuenta que el Epistolario nació de la necesidad de Catalina de Siena de dar respuesta a problemas concretos de sus devotos y de su tiempo. Personas diversas y de todas las categorías sociales e intelectuales se dirigieron a la monja de Siena en busca de consejo y ayuda. $\mathrm{Al}$ parecer, ya en vida de la santa, sus discípulos y secretarios sintieron la viva necesidad de recoger este material.

Su confesor, Raimundo de Capua, lo corrigió y matizó, y posiblemente lo trasladó en lengua latina. En 1492, 112 años después de la muerte de Catalina vieron la luz en la imprenta por prima vez, con el título de Epistole utili e divote de la beata e seraphica vergine sancta Caterina (impr. por G.F. Fontanesi, Bolonia). Poco después (en el año 1500), y en Venecia, Aldo Manuzio imprimió las Epistole devotissime de sancta Catharina da Siena, raccolte da Bartolomeo da Alzano da Bergamo ${ }^{10}$. Precisamente, de las diversas obras de Catalina de Siena se llegaron a realizar once ediciones, hasta el año 1500, hecho nada desdeñable, máxime si se tiene en cuenta que algunas salieron - como se ha dicho- de los prestigiosos talleres tipográficos de Aldo Manuzio".

El siglo de la imprenta se abrió con las cartas de santa Catalina, y la edición de Aldo Manuzio no sólo significó la aceptación de la santa en el mundo de la literatura, sino también un mensaje de reforma religiosa y moral dirigido a la Iglesia italiana $y$, en general, a toda Italia ${ }^{12}$.

\footnotetext{
${ }^{10}$ Esta prestigiosa edición italiana recogió tan sólo 219 cartas, mientras que las muy poco posteriores ediciones en lengua castellana - vid. apartado 3- reunieron ya 364 epístolas.

"Amedeo Quondam, La letteratura in tipografia, en "Letteratura italiana", diretta da A. Asor Rosa, vol. II, "Produzione e consumo". Einaudi, Torino, 1983, p. 596.

"Adriano ProsPeri, Lettere spirituali, en "Donne e fede. Santità e vita religiosa in Italia". a cura di Lucetta SCARAFFIA e Gabriella ZAN1, Laterza, Roma-Bari, 1994, pp. 227-251.

El prólogo de Aldo Manuzio, dirigido al cardenal Francesco Piccolomini, sobrino de difunto papa Pío II, refleja la actualidad de las cartas de Catalina de Siena. Afirma Manuzio: "Queste adunque si utile opera, benchè io non habbia familiarità alcuna con V.S.R., tamen l'ho dedicata a Quella per piu rispetti. Primo per eshortatione di devoti servi di Dio dell'ordine dei Predicatori dell'osservantia, per esser V.S.R. piena di bontà e santi costumi e vero cardinale della Chiesa di Dio. Poi, perchè ho stimato farli cosa grata dandoli uno tali fruto, uscito da una
} 
En cuanto a la autenticidad de las cartas, las ediciones recientes, como la de D. Umberto Meattini ${ }^{13}$, han fijado definitivamente su número (se consideran auténticas 381) y texto, aunque su contenido no respete literalmente la expresión salida de la boca o de la pluma de santa Catalina, sino que sus discípulos y compiladores prestaron atención no a la literalidad de las expresiones sino a los «profondi e soavi insegnamenti della loro madre e maestra e avevano di mira l'edificazione spirituale dei lettori, più che l'interesse storico» ${ }^{14}$. Además, muchas cartas fueron dictadas a los secreta$\operatorname{rios}^{15}$, con lo que las cartas, aunque auténticas, no pueden considerarse autógrafas. Los secretarios actúan de filtro y mediatizan las expresiones, eliminando expresiones vernáculas, como cuando recogen las cartas dictadas por la santa en estado de éxtasis.

En cuanto al orden numérico del Epistolario hay que destacar que fue obra de Niccolò Tommaseo, y fijado en la edición de 1860, bajo el título de Lettere de santa Caterina da Siena, orden y numeración que todavía continúan vigentes hasta la actualidad, y al que me he ceñido en las referencias que, en este texto, figuran entre corchetes.

fruttigena pianta della vostra inclita città di Siena. Item perchè havendo la detta vergine scritto di molte lettere a Sommi Pontifici e cardinali circa la reformatione della santa Chiesa e che si dispiegasse il confalone della Croce contra i pagani. E essendo state dette lettere fino a questi tempi (credo per volontà di Dio) quodam modo igcognite e ascoste, e se publichino hora che gl'infedeli sono in arme con stupendo essercito e apparato per mare e per terra con animo di distrugger la fede di Christo e già habbino cominciato a mandare ad essecutione il desiderio suo con grandissimo danno et strage di christiani, si può pensare che siano scritte più presto alli Pontifici de'tempi nostri che a quelli de allhora... E se non si provede presto, benche'l nome di Dio non possa perire e che'l braccio della Chiesa se s'indebilisce, non possa scavezzarsi; tamen siamo a grande pericolo non tocchi a noi che, oppresi dagli infideli, diventiamo tutti schiavi. Quod Deus avertat. Venetiis XIX sept. M.D.

${ }^{13}$ Santa Caterina Da Siena, Le lettere, a cura di D. Umberto MeatTini, Edizioni Paoline, Milano, 1987.

${ }^{14}$ Ibid., p. 35.

${ }^{15}$ Entre estos secretarios se cuentan por los menos cuatro personas, estrechamente vinculadas a la vida y actividad de la santa: Barduccio Canigiani, embajador florentino entre 1358-67, y posteriormente clérigo secular - tras la muerte de Catalina - sujeto a la dirección espiritual de fray Raymundo de Capua; Stefano Maconi (1347-1424), pariente lejano del papa Alejandro III, por línea materna (su madre era una Bandinelli), y que acabó de monje cartujo en Pavía; el tercero fue Ranieri di Landoccio Pagliaresi $(\uparrow 1406)$, miembro de una noble familia de Siena, culto y elegante prosista y poeta, se le considera autor de parte del Dialogo y de algunas cartas; el cuarto secretario fue Cristofano di Gano Guidini, notario de Vestrigona, a quien Catalina aconsejó sobre su futura esposa. Como amanuenses ocasionales figuran otros muchos personajes, entre ellos algunas mujeres como Francesca di Goro, viuda de Clemente di Goro, Alessandra Saracini o Giovanna dei Pazzi. 


\section{Protocolo de las cartas}

Catalina se dirige a sus corresponsales con tonos diversos según la condición de la receptora, en general :

"Carissima figliuola in Cristo dolce Gesù» a una meretriz [276] o a una monja [286]; «Dilettissima e carissima madre in Cristo dolce Gesù» [31] a una noble dama; o "Carissima figliuola in Cristo dolce Gesù» [113] dirigiéndose a otra joven miembro de la estirpe de los Salimbeni; «A voi, dilettissima e reverenda madre in Cristo Gesù» [145]; "A voi, dilettissima e reverendissima madre e suora in Cristo Gesù» [138]; «laudabile e carissima madre, madona la reina» [143]; dirigiéndose a la reina de Nápoles.

Saluda a su madré, monna Lapa, con fórmulas como «Carissima madre in Cristo dolce Gesù» [1] o «Carissima madre e figliuola in Cristo dolce Gesù» [117].

Las terciarias son saludadas como «Dilettissime e carissime figliuole mie» [108], o «Carissima e dolcissima suora mia in Cristo Gesù» [144]; mientras que las monjas o las prioras y abadesas de conventos y monasterios reciben tratos de «Carissima madre e figliuola in Cristo dolce Gesù» [75], «Dilettissime e carissime figliuole» [175], «Carissime suore in Cristo dolce Gesù» [215]. Trato similar reciben algunas damas no nobles: «Carissima figliuola in Cristo dolce Gesù» [300].

\section{LAS CARTAS A LAS MUJERES DE SU TIEMPO}

Catalina se dirigió en múltiples ocasiones a las mujeres de su tiempo. $\mathrm{Y}$ como "terciaria" que vive en el mundo y conoce el mundo y sus inquietudes y problemas, puede dirigirse a todo tipo de mujeres. Las diversas cartas conservadas demuestran la heterogeneidad social y su interés por "todas" las mujeres de su entorno, sin distinción de grupos, funciones o condiciones. Y todas podrán encontrar en el pensamiento y en la obra de Catalina algún lugar común, algun punto de referencia o de meditación.

Dada la variedad de las destinatarias, podemos agrupar las cartas en estos apartados: 


\section{a) Las cartas a las reinas}

Catalina se dirige, en su correspondencia, a Isabel de Hungría y a Juana I de Nápoles. La primera, Isabel, viuda del rey Carlos Roberto, gobernaba el reino en nombre de su hijo, el joven Luis I; la santa la incita a participar en la cruzada convocada por el pontífice (a quien califica de "il nostro Cristo in terra") con el fin de «fare il santo e dolce Passaggio sopra li cani malvagi Infedeli che posseggono il nostro e anco più» [145], en un momento en que los turcos asolaban las tierras de Dalmacia y Macedonia y amenazaban seriamente Sicilia.

El resto de cartas están dirigidas a Juana I de Anjou, reina de Nápoles. En tanto que los soberanos napolitanos ostentaban el título de "reyes de Jerusalén", Catalina pide a la reina que actúe en consecuencia, apoye activamente la cruzada promulgada por Gregorio XI y predicada por los dominicos (1375), a fin de que "così siate capo e ragione di questo passaggio, sì che quello santo luogo non sia posseduto più da quelli pessimi Infedeli, ma sia posseduto da'Cristiani onorevolmente, e da voi come cosa vostra” [143].

Pero otras cartas tienen como misión el reconducir la voluntad de Juana I en el conflicto del Cisma de Occidente - la reina de Nápoles apoyó en sus inicios al papa aviñonés-, y reintegrarla a la «obedienza e riverenzia dal padre nostro Urbano VI, il quale è veramente Cristo in terra, e ogni altro che venisse mentre ch'e' vive, non è papa, ma è peggio che Anticristo" [312], temática reiterada en la mayoría de las cartas dirigidas a la misma reina, llegando incluso a tonos de gran severidad y exigencia y recriminación ${ }^{16}$.

\footnotetext{
${ }^{16} \mathrm{La}$ traducción en lengua castellana, ordenada por el cardenal Cisneros, refleja con gran fuerza el estado de ánimo de Catalina de Siena: "Ay de mi! No esperéis el tiempo, que no sabéis si lo tendréis. No querráis que mis ojos ayan de derramar ríos de lágrimas sobre la pobre de vuestra ánima; la qual yo tengo por mía y lloro quando considero que es muerta por ser como lo es, apartada de su cabeça, persiguendo, no al Papa Urbano Sexto, sino a la verdad, y a vuestra fe, la qual esperava yo, segun que vos Madre, e hija mia me escrivisteis, que mediante el favor y gracia de Dios por vos avía de ser dilatada, y publicada entre los Infieles y esclarecida entre nosotros, defendiéndola de qualquiera que la quisiesse contaminar. Aora por mis pecados veo en vos todo lo contrario, por los malos consejos que os han sido dados, los quales vos como persona sin piedad para con vos misma, y para con vuestra salud aveis tomado. Yo veo que no ay criatura que pueda restituir vuestro daño; mas también à vos misma convendrá dar cuenta desto delante del sumo Juez. Esta offensa no procede de ignorancia porque vos sabéis la verdad, mas no sabéis volver atras, ni dexar lo començado, porque el cuchillo de la propia perversa voluntad os quita el saber, y el querer, dándoos a entender, que os sería vergüença lo que es grandíssima honra” (Epístola CCCXII, edic. 1698).
} 


\section{b) Cartas a las damas nobles}

Son diversas las damas nobles a las que se dirige Catalina de Siena. A través de la estrecha y mutua relación epistolar, se observa como las damas disputan la preeminencia para acoger a Catalina en sus palacios y salones, buscar sus sabios consejos o simplemente mantener una larga conversación con ella. A cambio, Catalina «entra in questi palazzi, bussa al loro cuore a affronta le più delicate situazioni domestiche, le esigenze dell'etica familiare, vagliandole su un piano in cui il particolare si rillaccia all'universale»' 17 .

Pero en ningún caso puede perderse de vista que el interés último de Catalina Benincasa radicaba en influir sobre los maridos o los hombres de un linaje y familia concretos a través de las esposas o de las mujeres más relevantes del grupo familiar ${ }^{18}$. Sin duda, uno de los casos más destacados es el de Raniera (o Niera), esposa de Gherardo Gambacorta de Pisa y madre de Lotto, futuro arzobispo de la misma ciudad: «Dite a Gherardo, che ora ch'è tempo accettabile, mentre che egli vive, che non dispregi questo sangue; però che non è sicuro quando debba morire, né quando debba vivere. Rechisi a vomitare il francidume de'peccati suoi per la bocca, confessandosi bene e diligentemente...» [155], y más tarde le añade «Benedite e confortate Gherardo in Cristo dolce Gesù. E dite a Gherardo, che io mi richiamerò a Cristo crocifisso di lui, perché egli non ha fatto quello che debbe fare ogni fedele cristiano. Dite che non aspetti l'ultimo dì della vita sua, però che non sa né quando né come» [224]. Catalina aconseja también en cuestiones de

«... Porque haziendo contra la verdad de la Iglesia, y contra el Papa Urbano Sexto, hazéis contra la verdad de Dios, y perdéis el fruto de la Sangre de Cristo, porque la Santa Iglesia es fundada sobre esta verdad. Sino queréis mirar a vuestra salud, tened respeto a los pueblos que os son encomendados, los quales avéis regido tanto tiempo con tanta diligencia y en tanta paz, y aora por hazer vos contra esta verdad, los veis desolados y destruidos y puestos en tanta guerra, matándose unos con otros como animales brutos, y no considerais vos, que de su pérdida, y por sus daños se disminuye vuestro Estado, y aun estáis obligada de dar cuenta a Dios de las ánimas que perecen, y que cuenta le podéis vos dar?"» (Epístola CCCXIII, edic. 1698).

${ }^{17}$ Umberto Meattini, Prefazione a S. Caterina da Siena, Le Lettere, Ediz. Paoline, Milano, 1987, p. 541 .

${ }^{18}$ Esto es especialmente detectable en los casos de las grandes familias de la Toscana. De todas formas, los grandes personajes de Siena y Florencia, destinatarios directos o indirectos de las observaciones de Catalina, no acabaron de fiarse de sus actuaciones o intenciones político-diplomáticas porqué con frecuencia recayó sobre Catalina la duda de estar al servicio de un bando rival. 
vida familiar, como el desposorio de los hijos, a este respecto es altamente representativa la respuesta a Niera sobre el futuro de su hijo: «Di quello che mi mandaste dicendo, d'allogare il vostro garzone, vi rispondo che voi attendiate non all'avere né a'grandi parentadi, ma solo alla virtù e alla buona condizione della fanciulla. Quando trovate questo, fatelo sicuramente» [224].

Otras destinatarias de la correspondencia cateriniana fueron las nobles Tora [194], Nella [151, 161, 167], Catarina y Orsola [153], todas ellas de Pisa. La primera, Tora, hija del Gambacorta, señor de Pisa, es receptora de un autentíco tratado de vida activa y provechosa, en la que destacaría estas palabras: «E guarda che tu non perda'l tempo tuo; imperocché molto più ti sarebbe richiesto ora che prima: ma sempre esercita il tempo o coll'orazione o colla lezione o con fare alcuna cosa manuale, acciocché tu non caggi nell'ozio; però che sarebbe pericolosa cosa» [262].

Entre las damas de Florencia mantuvo contactos con Constanza Canigiani, a raíz de haberse hospedado (1378) en su palacio [314], y Laudomia Strozzi [90], cuyo marido, Carlo delli Strozzi, fue uno de los apoyos más relevantes de la obra pacificadora de Catalina de Siena.

Entre las damas sienesas destacan en primer lugar Mitarella, esposa de Ludovico da Mogliano, que ostentó las más altas magistraturas de la ciudad y corrió serios peligros de perecer en medio de las turbulencias sociales de 1373-74, temores que Mitarella debió confesar a Catalina: «Mandastemi dicendo che, per lo caso che era occorso al Senatore (del quale mi pare che avete avuto grandissimo timore)... Suora mia, non temete le creature» [31]. También sienesa era la condesa Benedetta, hija de Giovanni d'Agnolino Salimbeni, a quien anima a la perfecta vida religiosa: "A te dico, figliuola mia, che se tu vorrai essere sposa vera del tuo Creatore, che tu esca della casa del padre tuo; e disponti de venire, quando il luogo sarà fatto; che già è cominciato, e fassi di forza: cioè il monastero di Santa Maria degli Angeli a Belcaro. Se tu'l farai, giungerai in terra di promissione» [112]; Bartolomea, esposa de Andrea Mei [71]; igualmente sienesa era Ludovica di Granello, a la que, en 1378, recuerda la obligación de dar limosna a los pobres: «Dovete essere dispensatrice dei poveri, voi e chi ne ha: perché e'poveri sono quelle mani...che ci fanno andare a vita eterna» [304].

Entre las mujeres milanesas figura la ambiciosa Beatriz della Scala, hija de Martino III de Verona, que se hacía llamar "regina", siendo sólo la esposa de Barnabó Visconti, señor de Milán. La orgullosa dama requirió la adulación de Catalina a través de un enviado "personal", a lo que la monja respondió con una epístola cargada de significado. De entrada la saluda con 
un «mia reverenda madre in Gesù Cristo», la insta a que influya sobre su marido, vuelva al redil de la vida cristiana y a la obediencia y sumisión al papa, y a ella la invita a despreciar las vilezas de la vida presente y a que ame y practique la virtud de la caridad [29].

Benedetta de'Belforti de Volterra recibe una auténtica apología de la virtud de la paciencia, mientras juega con la imagen de Cristo-médico, «venuto nel mondo per sanare le nostre infirmitadi» [68]. Benedetta era la esposa de Bocchino de Belforti, que acabaría asesinado tras una sublevación popular, y perdería también a sus hijos en circunstancias trágicas.

Génova también está representada con la figura de madona Orietta Scotta, que hospedó en su palacio de via Canneto al papa Gregorio XI y a Catalina [355].

Las damas de la nobleza de Nápoles también están representadas entre las receptoras de misivas. Una de ellas, Laura o Hilaria, esposa de Francesco Caracciolo [352], otras, diversas mujeres reunidas en cenáculo, a quienes insta a la plegaria continuada, y las instruye sobre los diversos tipos de oración (llamado "cibo angelico") que sintetiza en tres: la oración continua, la vocal y la mental [353]. La carta a una dama de la corte de Juana I de Nápoles la estimula al temor de Dios y al rechazo al temor servil [361]. Finalmente, la epístola a madonna Pentella, esposa de un adúltero, recoge la exaltación de las virtudes de la paciencia, fortaleza, templanza y la caridad [454].

\section{c) Cartas a las mujeres de su entorno familiar}

Sin duda alguna las más notorias y representativas son las que Catalina dirigió a su madre, Lapa di Puccio di Piagente, hija de Muzio Piagente, poeta de cierta notoriedad, y esposa - obviamente- de Jacopo Benincasa, padres ambos de santa Catalina. Lapa fue la viva representación de la madre que se desvela por sus hijos, y entre todos ellos se desvela por su hija Catalina. A monna Lapa, Catalina le recuerda, desde Aviñón, que su situación es similar a la de la Virgen María tras la muerte de Jesús: «Or vi confortate per amore de Cristo crocifisso, e non crediate d'essere abbandonata né da Dio né da me. Anco, sarete consolata, e riceverete piena consolazione; e non è tanta stata la pena quanto sarà maggiore il diletto. Tosto ne verremo, per la grazia di Dio...» [240], y le hace reiterativas llamadas a las virtudes de la paciencia y la generosidad [6]. Las sobrinas, sobre todo las hijas de su hermano Bartolomeo, fueron las destinatarias de diversas cartas 
llenas de ternura [23, 26], en especial la que tomó los hábitos, sin vocación, en el convento de Santa Agnese de Montepulciano, y a la que repite su exégesis sobre la oración y sus diversas tipologías, descrita anteriormente, calificada ahora de «cibo che, mentre siamo peregrini in questa vita, tira a sé l'odore delle vere e reali virtù».

\section{d) Cartas dirigidas a sus discipulas}

Catalina de Siena contaba con tres mujeres entre sus discípulos. Consta que siempre tuvo a su lado una o dos mantellate o terciarias. Se cuenta entre las predilectas a Lisa Colombini, su cuñada; a Alessia Saracini, destinataria de cinco cartas $[49,119,271,277,286,126]$, y en cuya casa Catalina se alojó en múltiples ocasiones, incluso durante semanas enteras. Y no menos importante fue la fiel escribana y secretaria Giovanna Pazza, miembro de la noble familia sienesa de los Cinughi de'Pazzi, trasladada de Florencia a Siena a mediados del siglo XIII.

\section{e) Cartas dirigidas a las terciarias dominicas o "mantellate"}

El orden tercero de los dominicos acogía, como es de sobras conocido, a hombres y mujeres que, sin renunciar a vivir en el mundo, aspiraban a cotas de mayor perfección siguiendo unas normas mínimas de conducta. En la ciudad de Siena, un grupo de mujeres, miembros del tercer orden dominicano, se distinguían por sus hábitos y por la larga mantilla de color negro que cubría un vestido simple de color blanco ${ }^{19}$. Estas mujeres seguían una regla y unas prácticas religiosas, tenían un director espiritual miembro de la orden. La misma Catalina entró a formar parte de este grupo a la edad de 16 años - a pesar de la resistencia de su madre Lapa-, en la capilla delle Volte de la iglesia de Santo Domenico de Siena (entre 1363-64). De ahí la intensa relación y confianza que Catalina depositó a lo largo de su vida en este grupo de religiosas, algunas de las cuales figuran entre el grupo de sus más directas discípulas. Además de estas, ya reseñadas, el grupo de las destinatarias de cartas incluye a personajes sieneses como Francesca y Ginoccia Tolomei [81], Giovanna di Capo [108, 132], Caterina dello

\footnotetext{
${ }^{19}$ Nótese que se trata de los mismos colores, blanco y negro, característicos del hábito de los dominicos.
} 
Spedaluccio [118, 214], Agnese de Malevolti [38, 53, 61], de cuyas epístolas se refleja el ambiente apostólico y la vivencia misionaria de las mantellate. Lucca, Orvieto, Fiesole o Pisa son otros centros no menos importantes de comunidades de terciarias con las que Catalina mantuvo alguna correspondencia $[97,144,153,166,65,213]$. Todas ellas se caracterizan por el apostolado de oración y penitencia, en la línea que la misma santa comenta en sus tratados ascéticos, o refleja en sus oraciones.

\section{f) Cartas dirigidas a monjas o comunidades monásticas del centro de Italia}

Diversas y variadas son las cartas de Catalina dirigidas a monjas, tanto de la orden benedictina como dominicas. A través de ellas la autora revive su ideal monástico, y con sus ejemplos y sus consejos remarcaba las normas de vida de comunidades nuevas o de comunidades ya consolidadas. Es más, Catalina no dejó de lamentar [296] las numerosas dificultades para sacar adelante una auténtica reforma del monaquismo $0^{20}$ a lo largo de la segunda mitad del siglo XIV, y la superación de decadentes normas jurídicas en total desacuerdo con la auténtica imitación de Cristo ${ }^{21}$.

No siempre es fácil identificar la orden de cada una de las abadesas o comunidades receptoras. Entre las benedictinas de Siena deben contarse sor Constanza y sor Magdalena, del monasterio de Sant'Abondio [73, 220], monasterio íntimamente relacionado con el beato Colombini, que tuvo que exiliarse de la república a causa de sus propuestas de "comunismo cristiano"22. El monasterio de san Pietro a Monticelli (Florencia) [79] y el de

\footnotetext{
${ }^{20}$ Sobre la nueva concepción del monaquismo en Catalina. es interesante la observación de Claudio LEONARDI, Caterina da Siena: mistica e profetessa, en "Atti del Simposio Internazionale Cateriniano-Bernardiano (Siena, 17-20 aprile 1980)", Accademia Senese degli Intronati. Siena, 1982, p. 170: "la sua cella monastica, ella dice, non è di pietra o di legno, è una cella interiore, è una condizione ontologica, è l'inabitazione di Dio nell'uomo. Il monachesimo sopravvive, dopo di lei, nella Chiesa, ma come forza storico-ecclesiale è fïnito, o almeno il suo segno dovrà mutare: dall’ascesi...e culmina nella contemplazione di Dio, si passa all’imitazione del Cristo e alla conversione del mondo».

${ }^{21}$ Tal es el caso, por ejemplo, del rechazo de los hijos ilegitimos a acceder a la plenitud de la vida monástica. Vid. Giorgio PICASSO OSB, Santa Caterina e il mondo monastico del suo tempo, en "Atti del Simposio Internazionale Cateriniano-Bernardiano (Siena, 17-20 aprile 1980)", p. 277.

22Umberto Meattini, Prefazione a Santa Catalina da Siena, Le Lettere, p. 1029; Guido BALDASARI, Letteratura devota, edificante e morale, en "Storia della Letteratura Italiana", vol. II, "Il Trecento", Salerno Edit., Roma, 1995, pp. 218-222.
} 
Santo Stefano fuori Porta, en Pisa [182, 188, 221], así como el de Monte San Savino, en Arezzo [75], constituyen otros ejemplos a destacar.

Los monasterios de agustinas figuran también entre las comunidades favorecidas con alguna atención epistolar de Catalina. Precisamente el cenobio de Santa Marta, en San Marco (Siena) [30, 180]. Las comunidades de dominicas destacan por su mayor número, y entre ellos el monasterio de Sant'Agnese de Montepulciano, donde profesó su sobrina sor Eugenia [54, $58,336,381]$.

\section{g) Cartas a diversas mujeres o grupos de mujeres de procedencia y ocupaciones muy diversas}

Dada la variedad de las destinatarias señalaré tan sólo algunos ejemplos. Entre los más significativos resaltan las tres mujeres florentinas [82], a quienes aconseja paciencia, caridad y la sublimación de las penas, «e fuggirete le conversazioni, e diletteretevi della solitudine, e non presumerete di voi», todo un programa de vida, al que añade poco después la necesidad de contar con un único guía espiritual, «il quale vi consigli squietamente, e quello seguitate».

Franceschina y Caterina, Melina Balbani, y Bartolomea Salvatico, todas ellas mujeres de Lucca $[162,163,164,165]$, a las que da consejos múltiples sobre la educación de los hijos, la relación con los maridos y, sobre todo, la vida espiritual como mujeres devotas de su tiempo. La búsqueda del justo equilibrio en la práctica de la penitencia, sin extralimitaciones ni exageraciones, será otro de los temas caros a Catalina y objeto de observaciones a algunas mujeres, como la penitente Agnese da Toscanella, a quien recuerda: "Alcuna volta sono molti che ricevono inganno nella penitenza... la penitenza si deve pigliare per strumento, e usare per augmentare la virtù, e per mortificare il corpo, ma non per principale affetto...» [340].

Pero de todas estas cartas destaca la 276, dirigida a una meretriz, a petición de su hermano: Le propone el modelo de la "dolce e innamorata" Magdalena que siguió a Cristo, y a la que le fueron perdonados sus pecados a cambio de no volver a pecar. Describe sutilmente la condición de la meretriz, para reprenderla: «Or non vedi tu che tu se'colei che ti dài nelle mani degli uomini a fare strazio, scherni, e scempio delle carni tue? or non vedi tu che se'amata e ami d'uno amore mercennaio che ti dà morte?» 
Igualmente singular es la epístola dirigida a una murmuradora [307], a quien advierte «come è torta la loro via, che, essendo ciechi, vogliono giudicare la luce! ... Oh superbia umana! E come non si vergogna la creatura di volere tollere l'officio di mano al suo Creatore? Che alla creatura sta d'aspettare d'essere giudicata, e non di giudicare». También esta epístola - como la anterior - puede considerarse una de las más directas, que ataja el problema de la receptora, de ahí que la epístola termine con una severa recomendación: «Ponete fine e termine oggimai a ogni vostra passione, e non vogliate prestare l'orecchie in udire quello che non dovete: ma, come persona che non vuole la dannazione dell'anima sua, attenetevi alla verità; e non vogliate scandalizzarvi così di leggiero».

\section{LA RECEPCIÓN/TRADUCCIÓN DE LAS EPÍSTOLAS EN ESPAÑA}

La primera edición del texto de las Epístolas, traducidas en lengua castellana, se llevó a cabo en 1512, en Alcalá de Henares, por el impresor Arnaldo Guillén de Brocar, y bajo las órdenes del cardenal Cisneros. Es posible que se tomaran como modelo las dos primeras ediciones italianas de 1492 y 1500 , aunque es posible que llegaran a Alcalá a través de copias manuscritas. De todas formas conviene tener presente que el cardenal Cisneros mandó traducir muchas más epístolas de las contenidas en la primera edición de Venecia, hasta un total de 364 (sobre las 381 reconocidas en la actualidad como auténticas), aunque ello no se especifique claramente en el título: Obra de las epístolas y oraciones de la bien aventurada virgen sancta Catherina de Sena dela orden de los predicadores. Las quales fueron traducidas del toscano en nuestra lengua castellana por mandado del muy Illustre y Reverendíssimo señor el Cardenal Despaña Arçobispo della Sancta Yglesia de Toledo, etc.

Esta edición va acompañada - como se indica en su prólogo- de 25 oraciones de la santa y de un breve reflejo de la vida de la virgen Catalina «la qual escrivió el venerable religioso fray Estevan de Sena prior del monasterio de Sancta María de Gracia de la cibdad de Pavía de la orden de la Cartuxa, famyliar de la dicha virgen ${ }^{23}$ a fray Thomás de Antonio de la

\footnotetext{
${ }^{23}$ Se trata de uno de los secretarios de la santa, Stefano Maconi que, tras la muerte de Catalina, acabó entrando en la orden de los cartujos por indicación de la misma santa, llegando al cargo de prior de la cartuja de Pontignano en Pavía. Catalina se dirigió a él con estas
} 
orden de predicadores, en la qual trata brevemente algunas cosas de su vida [de Catalina] y conversación y de algunos milagros».

La segunda edición conocida apareció en Barcelona, el 1698, y reúne igualmente Epístolas y Oraciones de la santa, bajo el título de: Ramillete de epístolas, y oraciones celestiales para fecundar todo género de Espíritus. Nacido en el ameno jardín de las virtudes todas el coraçón de la Mystica Doctora, y Seráfica Virgen Santa Cathalina de Sena de la Sagrada Orden de Predicadores. Que mandó traducir a la lengua castellana de la toscana propia de la Santa, el Eminentíssimo Señor Don Fr. Francisco Ximénez de Cisneros, por la Divina Miseración Cardenal, y Canciller Mayor de Castilla, etc. Dedicase y conságrase a la misma Santa. Año 1698. Con licencia, en Barcelona. A costa de Ivan Cassañes y Iaume Suriá, libreros.

En esta nueva edición se tomó como referente la anterior, impresa en Alcalá de Henares, de 1512, con igual número de epístolas, aunque, como indica el prólogo anónimo, "Verdad es que en esta última impresión de parecer de hombres doctos, se han puesto algunas voces, términos o palabras al uso razonable de la ortografía moderna y corriente, como más clara, más pulida, más graciosa y más elegante...» y todavía aclara el prologuista: «Pero bolviendo al Libro de nuestras Cartas te digo del, que es el non plus Ultra, de todas sus Obras, en el qual la Santa excediéndose a sí misma remonta el buelo de su delicada pluma sobre todo quanto escrivió, y dictó, pues del todo se pierde de vista a los ojos más perspicaces.... Este excesso que tienen las Cartas familiares de los Santos sobre todo lo demás que escrivieron notó el Ilustríssimo, y Reverendíssimo Señor Don Iuan de Palafox, y Mendoza Obispo de Osma en aquellas notas de oro, y comento precioso que hizo sobre las Cartas de la Seraphyca, y Mystica Doctora Santa Teresa de Jesús...»

Ambas ediciones en lengua castellana, las dos conocidas hasta la fecha $^{24}$, contienen el mismo número de cartas, pero no conservan la misma numeración ni orden, y han sido calificadas de «mal curate per madornali

palabras: «A te poi in virtù della santa obbedienza comando in nome di Dio di entrare nell'ordine dei Certosini, perché ad esso Dio ti ha chiamato e scelto" Cit. en Giorgio PICASSO, OSB, Santa Caterina e il mondo monastico del suo tempo, p. 278.

${ }^{24}$ Hay traducciones parciales en lengua castellana, obra de Giselda ZANI; SANTA CATALINA DE Siena, Sesenta cartas políticas, Ed. Losada, Buenos Aires, 1950; y también en lengua catalana, obra de Tomás Garcés, vid.: SANTA CaTalina DE SIEna, Cartes i pensaments, Ed. Barcino, Barcelona, 1927. 
errori di vocaboli; sensi cambiati, senza parlare di come era stato bistrattato il dolce idioma senese ${ }^{25}$.

Quisiera insistir en el hecho usual que la traducción de las epístolas conlleve también la popularización y exposición de la vida y milagros de la santa, ya sea como complemento de las obras - tal ocurre en la traducción ordenada por Cisneros - o coincide su expansión con las traducciones de las biografías de la santa, como la de fray Raimundo de Capua ${ }^{26}$ en lengua castellana, y la traducción en lengua catalana ${ }^{27}$ que se llevó a cabo en Valencia, en 1511 - un año antes de la edición castellana de las epístolas ordenada por el cardenal Cisneros-; la edición valenciana va acompañada de una serie de poemas de autores relevantes como Narcís Vinyoles, Vicent Ferrandis o Pere Martí ${ }^{28}$. En cualquier caso es una forma eficaz de potenciar y popularizar la fama de santidad y el modelo de vida propuesto

${ }^{25}$ Santa Caterina da Siena, Le lettere, a cura di D. Umberto MEattini. Edizioni Paoline, Milano, 1987, p. 42.

${ }^{26}$ RAIMUNDO DE CAPUA, beato, La vida de la seraphica sancta Catherina de Sena, Impr. Pedro Lasso, Salamanca, 1588; RAIMUNDO DE CAPUA, beato, Vida y milagros de la bienaventurada sancta Catherina de Sena, trasladada de latín en castellano por Antonio de la Peña, Impr. Manuel Rodriguez y Juan Piza, Mallorca, 1617.

${ }^{27}$ Me refiero a la edición valenciana salida de la imprenta de Joan Joffre de Briansó, en 1511, con la traducción de fray Tomás de Vesach, bajo el título: La vida dela serìphica Catherina de Sena ara novament per un devot affectat religiòs della complidament arromançada: $e$ de moltes istories istoriada. E no res menys per un reverent mestre en sacra theologia molt bé e diligentment corregida y examinada.

El prólogo no tiene desperdicio, ni en la dedicatòria ni en la exposición de la intencionalidad de la obra, que se considera auténtico modelo de conducta y de piedad, con plena vigencia hasta el siglo XVIII (Vid. Antoni Comas, Historia de la Literatura Catalana. Part Moderna, vol. VI, Ariel, Barcelona, 1980, p. 34).

"...e pensant que tant devotíssima legenda com aquesta, en pochs lochs se troba; de hon de necessitat se segueix, que la sanctetat e perfecció de aquesta sancta verge està com la lum en la lanterna, o la pedra preciosa dins en lo sudari, contínuament amagada: per ço tant per la devoció que a la dita sancta porte, quant encara perquè dita istòria puga ésser largament e difusa comunicada a tots aquells, e aquelles qui la volran la he feta emprenptar en nostra lengua valenciana: No curant empero de servar estil poètich ni modo artizat en lo arromançar, simó planament e grossera segons lo meu modo comú de parlar. E aço he fet tant per esquivar semblants modos los quals per als religiosos no satisfan, quant encara perque moltes vegades en coses devotes, y espirituals fan perdre la sentència, e la intel-ligencia de la istòria especialment entre indoctes, e ignorants segons són per maior part les dones, e altres persones semblants, per amor de les quals he volgut pendre principalment aprés dur aquesta fatiga, e treball certificant que en la present istöria per mi arromançada res de nou cap que mude sentència no y he affïgit ni levat: sinó felment de latí en romanç trelladant».

28 "Obres fetes en lahor de la seràthica santa Catherina de Sema em el seu sagrat monestir de les monges de la insigne ciutat de Valencia per diversos trobadors narrades lo dia de sent Miquel del any M.D.XI. Essent iutges lo reverent frare Balthasar Sorio mestre en sacra Theologia e vicari general del frares observants del orde de preycadós. E lo reverent senyor canonge Fira y lo noble don Francisco Fenollet". 
por santa Catalina. El "curriculum vitae" propuesto y establecido por fray Raimundo de Capua reúne la ventaja, nada desdeñable, de presentar un nuevo modelo de santidad, en el que tienen cabida las mujeres del ámbito secular: madres, doncellas, viudas, reinas, damas de corte, e incluso mujeres de vida licenciosa, sin que el nivel social fuera óbice para ascender a más altos niveles de perfección.

Además; la febril actividad de la santa, puesta de relieve tanto en la biografía como en las epístolas, permite presentarla como modelo, no ya de santidad, sino también de mujer cristiana que actúa en el mundo - no se olvide su condición de "terciaria" dominica- y se implica en los problemas religiosos y sociales de su época. Y a la larga, su modelo de santidad visionaria, acabaría por ser la referencia más significativa de numerosas monjas carismáticas, las "santas vivas" de cortes renacentistas y de numerosas reformadoras místicas ${ }^{29}$. Es más, la influencia de la obra y de la vivencia religiosa de santa Catalina fue importantísima en la literatura epistolar del Renacimiento y del Barroco, y lo que fue un fenómeno de excepción en el Trecento y Quattrocento - la de la mujer que escribe cartas, fuere reina, noble o santa- comenzó a abrirse camino y a consolidarse dentro del panorama de la literatura de aquellas épocas, de la que emergerían el modelo hispánico de santa Teresa de Jesús o los italianos de Caterina de Genova, Camilla Battista Varano y, sobre todo, de Maria Maddalena de'Pazzi ${ }^{30}$.

\section{RÉSUMÉ}

Analyse des lettres écrites par Catherine de Sienne à plusieurs femmes de son époque: des reines, des religieuses, des dames de la noblesse, des bourgeoises et même des courtisanes. On souligne ici les caractéristiques générales de cette correspondance ainsi que les rares aspects personnels relevés dans les lettres les plus significatives. Les deux éditions en langue castillane, l'une de 1512 et l'autre de 1698, montrent l'importance du recueil de lettres en tant qu'oeuvre littéraire et religieuse.

\footnotetext{
${ }^{29}$ Sara F. MATTEWS GRIEGO, Modelli di santità femminile nell'Italia del Rinascimento $e$ Controriforma, en "Donne e fede. Santità e vita religiosa in Italia", a cura di Lucetta SCARAFFIA e Gabriella ZANI, Laterza, Roma-Bari, 1997, p. 317.

${ }^{30}$ Maria ZaNCAN, La donna, en "Letteratura italiana", diretta da Alberto Asor Rosa, vol. V, "Le questioni", Einaudi, Torino, 1986, pp. 809-811; Maria Luisa DoGLIO, Lettera e Donna. Scrittura epistolare al femminile tra Quattro e Cinquecento, Bulzoni, Roma, 1993, d.p.
} 


\section{SUMMARY}

A study of the letters written by St. Catherine of Sienna to women of her epoch including queens, nuns, noblewomen, members of the middle classes and even prostitutes. The general characteristics of this correspondence are emphasised as are the scant personal aspects which feature in the most important ones. The two editions in Castillian, in 1512 and in 1698 , show the importance of the letters, both as a literary and a religious work. 